\title{
SLIDING CONTACT LOADING ENHANCES THE TENSILE PROPERTIES OF MESENCHYMAL STEM CELL-SEEDED HYDROGELS
}

\author{
Alice H. Huang ${ }^{1,2}$, Brendon M. Baker ${ }^{1,2}$, Gerard A. Ateshian ${ }^{3}$ and Robert L. Mauck ${ }^{1,2, *}$ \\ ${ }^{1}$ McKay Orthopaedic Research Laboratory, Department of Orthopaedic Surgery, University of Pennsylvania, \\ Philadelphia, PA USA \\ ${ }^{2}$ Department of Bioengineering, University of Pennsylvania, Philadelphia, PA, USA \\ ${ }^{3}$ Departments of Mechanical and Biomedical Engineering, Columbia University, New York, NY, USA
}

\begin{abstract}
The primary goal of cartilage tissue engineering is to recapitulate the functional properties and structural features of native articular cartilage. While there has been some success in generating near-native compressive properties, the tensile properties of cell-seeded constructs remain poor, and key features of cartilage, including inhomogeneity and anisotropy, are generally absent in these engineered constructs. Therefore, in an attempt to instill these hallmark properties of cartilage in engineered cell-seeded constructs, we designed and characterized a novel sliding contact bioreactor to recapitulate the mechanical stimuli arising from physiologic joint loading (two contacting cartilage layers). Finite element modeling of this bioreactor system showed that tensile strains were direction-dependent, while both tensile strains and fluid motion were depth-dependent and highest in the region closest to the contact surface. Short-term sliding contact of mesenchymal stem cell (MSC)seeded agarose improved chondrogenic gene expression in a manner dependent on both the axial strain applied and transforming growth factor- $\beta$ supplementation. Using the optimized loading parameters derived from these short-term studies, long-term sliding contact was applied to MSC-seeded agarose constructs for $21 \mathrm{~d}$. After $21 \mathrm{~d}$, sliding contact significantly improved the tensile properties of MSC-seeded constructs and elicited alterations in type II collagen and proteoglycan accumulation as a function of depth; staining for these matrix molecules showed intense localization in the surface regions. These findings point to the potential of sliding contact to produce engineered cartilage constructs that begin to recapitulate the complex mechanical features of the native tissue.
\end{abstract}

Keywords: Cartilage; tissue engineering; mesenchymal stem cells; chondrogenesis; mechanical stimulation.

*Address for correspondence:

Robert L. Mauck, Ph.D.

McKay Orthopaedic Research Laboratory

Department of Orthopaedic Surgery

University of Pennsylvania

$36^{\text {th }}$ Street and Hamilton Walk

Philadelphia, PA 19104, USA

Telephone Number: 215 898-3294

FAX Number: 215 573-2133

Email: lemauck@mail.med.upenn.edu

\section{Introduction}

The load-bearing capacity of articular cartilage is enabled by a set of unique mechanical and biochemical properties that are both inhomogeneous (depth-dependent) and anisotropic (direction-dependent). The solid extracellular matrix (ECM) of cartilage is primarily composed of proteoglycans enmeshed within a type II collagen network (Mankin et al., 1994; Muir, 1980). Together, these matrix molecules govern the compressive and tensile properties of cartilage, which resist the stresses arising from joint movement. While the equilibrium compressive modulus is high (0.2-1.4 MPa) for a soft tissue (Ateshian et al., 1997; Athanasiou et al., 1991; Mow et al., 1980) and inhomogeneous through the depth (lowest in the superficial region, highest in the deep zones), the tensile modulus (1-30 MPa) is an order of magnitude higher and is critical to tissue function (Akizuki et al., 1986; Roth and Mow, 1980; Woo et al., 1976). The organization and orientation of collagen fibers dictate tensile properties, which are highest in the superficial zone (the region of highest collagen content) and in the split-line direction (parallel to fiber orientation) (Mow and Guo, 2002; Woo et al., 1976). This disparity between the equilibrium compressive and tensile properties enhances the ability of cartilage to resist physiologic cyclic loading (Cohen et al., 1998; Soltz and Ateshian, 2000).

The development of these depth- and directiondependent properties is directed in part by mechanical forces; mechanical loading with motion induces remodeling of the immature matrix (Archer et al., 2003), leading to increases in compressive and tensile properties (Athanasiou et al., 2000; Kempson, 1991; Kempson, 1982). The increases in tensile properties correlate with increases in collagen content and cross-linking (Charlebois et al., 2004; Williamson et al., 2003a; Williamson et al., 2001). In the absence of this loading environment, the tensile properties of embryonic and juvenile cartilage explants decrease, in conjunction with decreases in collagen and cross-link density (Williamson et al., 2003c). These findings suggest that the mechanical demands placed on cartilage lead to rapid changes in collagen content and organization, allowing the tissue to achieve its mature load bearing function.

While the healthy tissue is able to withstand the high stresses generated by joint loading, this load bearing capacity is often permanently lost with injury or disease, as the healing potential of degenerated cartilage is limited. To meet the growing demands for cell-based therapies, tissue engineering has therefore emerged as one promising strategy. Mesenchymal stem cells (MSCs) are an attractive cell type for these applications, as they can undergo 
chondrogenesis within many biomaterial systems and synthesize a cartilaginous matrix (Erickson et al., 2009; Huang et al., 2010b; Huang et al., 2004b). Under defined chondrogenic media conditions, the equilibrium compressive properties of MSC-seeded constructs can reach as high as $250 \mathrm{kPa}$ (Huang et al., 2009). While the compressive properties in engineered constructs are often evaluated, few studies consider tensile properties as a primary design criterion, despite the importance of tensile properties to articular cartilage function. In previous work, we charted the tensile properties of MSC-seeded hydrogels in free-swelling culture and found that while tensile properties initially increased with time in culture as matrix was deposited, within a few weeks tensile properties plateaued to levels far below those of the native tissue (Huang et al., 2008). Biochemical evaluation showed poor collagen deposition and little collagen organization.

Therefore, generating MSC-based constructs with the mechanical complexity and integrity of cartilage remains a challenge; not only are the properties of MSC-seeded hydrogels consistently lower than those of the native tissue (Huang et al., 2010b; Mauck et al., 2006), but the structural organization of articular cartilage is completely absent. As mechanical stimulation is critical to cartilage development and maturation, bioreactor systems that simulate the native mechanical environment of cartilage may bridge these functional disparities (Grad et al., 2011). Indeed, dynamic axial compression applied to MSC-based engineered cartilage enhanced compressive properties, though collagen content remained low (Huang et al., 2010a). While promising, these studies were not designed to generate either depth-dependence or constructs with improved tensile properties. Therefore, to better enhance construct properties and instill depth- and direction-dependent properties, a loading modality more representative of the native joint environment may be required.

The design of such a system is aided by numerous studies of cartilage mechanics during joint movement. In the native joint environment, including that of synovial joints such as the knee and ball-and-socket joints such as the hip, physiologic loading can be best described as a sliding contact between two cartilage layers (Ateshian and Hung, 2003). Loading is characterized by a moving contact area across curved surfaces and the applied load is largely borne by fluid pressurization (Ateshian and Wang, 1995; Caligaris and Ateshian, 2008). Fluid pressurization arises within the contact region and shields the solid matrix from excessive deformation (Soltz and Ateshian, 1998). As fluid is displaced by loading, it travels via the shortest path toward the contact periphery where pressure is low. Theoretical models of frictionless rolling contact show that resistance to fluid motion increases with increased joint congruency (due to longer fluid travel paths), leading to increased fluid load support (Ateshian and Wang, 1995). Recapitulation of these mechanical events in vitro may improve construct properties and instill a cartilage-like architecture.

To date, relatively few studies have examined the effects of sliding contact on the functional growth of engineered constructs. In one study, a migrating contact applied to adult canine chondrocyte-seeded agarose disks improved the equilibrium compressive properties, though no differences were observed in biochemical content or spatial distribution of matrix (Bian et al., 2010a). Related studies applying rolling contact (Stoddart et al., 2006) or compression and shear (Grad et al., 2006a; Grad et al., 2005; Grad et al., 2006 b) to chondrocyte-seeded polyurethane sponges showed that these loading modalities modulated chondrogenic gene expression and proteoglycan synthesis, however mechanical properties were not assessed. In one very recent study (Grad et al., 2012), compression and shear applied over three weeks, increased the indentation stiffness (assessed by AFM) and decreased the friction coefficient of the surface layer of engineered chondrocyte-based constructs. Compression and shear in these studies were applied using an oscillating spherical hip ball and the contact area remained constant with loading, with shear loading superimposed on dynamic compressive loading. Using this oscillating-ball bioreactor, recent studies have also examined the effect of dynamic compression and shear on MSC-seeded fibrin-polyurethane sponges. While MSC gene expression was modulated by loading, the GAG content of constructs was not affected ( $\mathrm{Li}$ et al., 2009a; Li et al., 2009b). Changes in gene expression were dependent on the presence and concentration of transforming growth factor- $\beta 1$ (TGF- $\beta 1$ ) during loading (Li et al., 2009a). Although these findings illustrate the potential of mechanical signals resulting from compression and shear to modulate MSC behavior in 3D cultures, the physical signals imparted by the loading system on the MSCs seeded within these composite biomaterials was not characterized. In addition, none of the studies mentioned above was designed to examine the effect of loading on developing tensile properties or collagen content.

To further this line of inquiry, we developed and characterized a new sliding contact bioreactor system that recapitulates the mechanical stimuli arising from joint motion (two contacting cartilage layers). Finite element modeling (FEM) analysis of sliding contact on agarose (a well-characterized biomaterial) (Albro et al., 2008; Chahine et al., 2009; Mauck et al., 2003) showed that tensile strains (parallel to the sliding direction) and fluid efflux/influx were depth-dependent and highest in the region closest to the construct surface. Using this bioreactor system, we demonstrated improved expression of chondrogenic genes with short-term sliding contact of MSC-seeded agarose; these changes in gene expression were dependent on both axial strain magnitude and TGF- $\beta$ supplementation. We then applied long-term sliding contact to MSC-seeded agarose constructs using the optimized parameters previously determined from short-term studies, and showed improved tensile properties and depth-dependent matrix remodeling with sliding contact.

\section{Materials and Methods}

\section{Sliding contact bioreactor}

A custom, displacement controlled bioreactor (Fig. 1) was constructed to apply sliding contact (SLC) to cellseeded hydrogel strips. The bioreactor consisted of an array of spherical polytetrafluoroethylene (PTFE) indenters ( $\varnothing 25 \mathrm{~mm}$ ) cut to $10 \mathrm{~mm}$ width and length, a lid to maintain sterility, removable polysulfone trays, and a motorized 

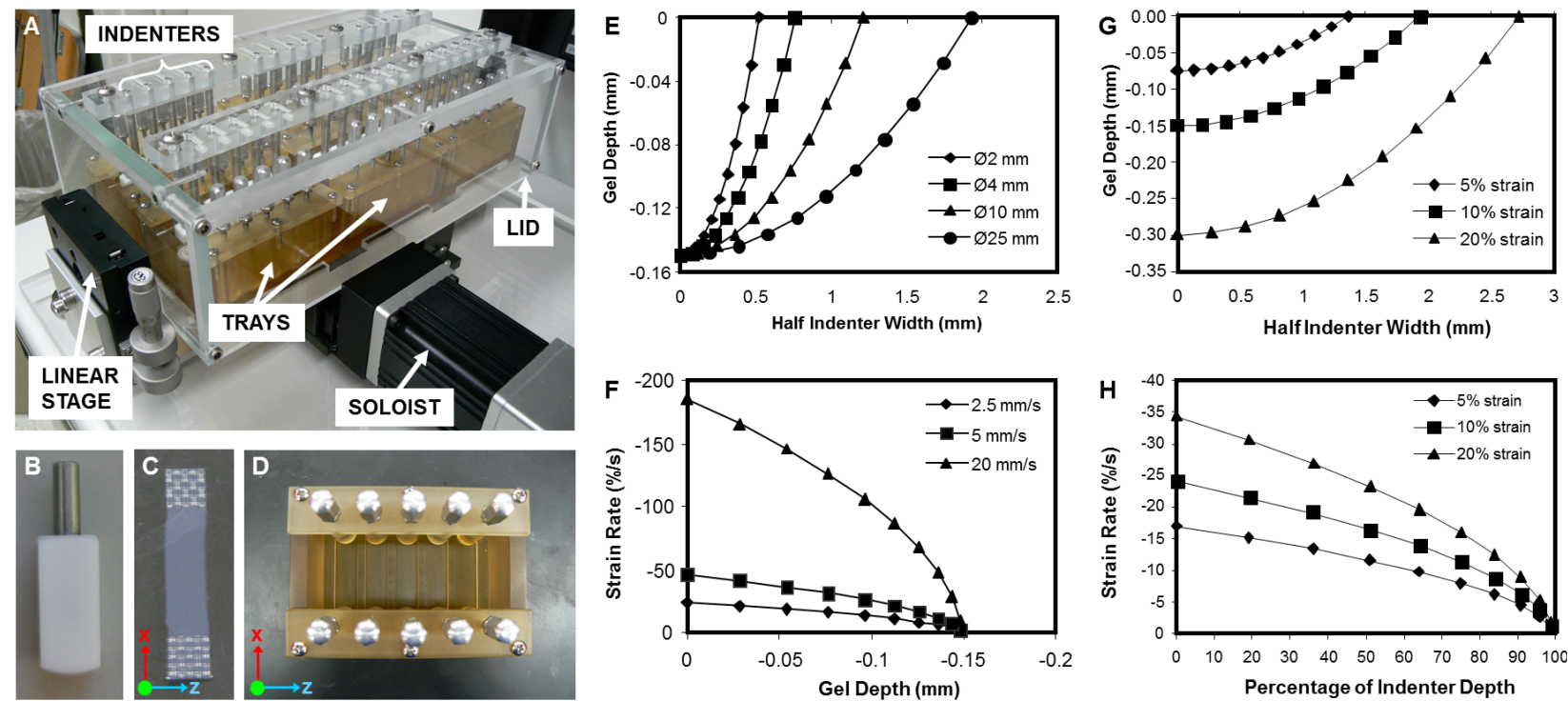

Fig. 1. A displacement-controlled bioreactor designed to apply sliding contact (SLC) to engineered cartilage constructs. (A) The bioreactor consisted of spherical indenters, a lid and removable trays. Axial and sliding displacements were controlled by linear stages and a stepper motor (Soloist), respectively. (B) SLC was applied using spherical indenters (original diameter: $\varnothing 25 \mathrm{~mm}$, sectioned to decrease chamber size). (C) Cell-seeded hydrogel strips were cast with nylon meshes on either end to enable fixation into bioreactor trays. (D) Strips were housed in removable trays and held in place by posts, with up to five strips per tray. (E, G) Indenter displacement and (F, H) axial strain rate were calculated for a range of diameter sizes and axial displacements.

motion control system (Soloist, Aerotech, Pittsburgh, PA, USA) to regulate sliding displacement magnitude and velocity (x-direction). Axial displacement (y-direction) was controlled manually with ball-bearing linear stages and actuators (Newport, Irvine, CA, USA). Strips were maintained and loaded in rectangular wells within trays and held in place by clamps on either end, with up to 5 strips per tray. For all experiments, a reciprocating sliding displacement was applied.

\section{Finite element analyses}

A finite element model was generated using experimentallydetermined properties and geometries of day 21 MSCseeded constructs. A biphasic material was used with intrinsically incompressible fluid and solid phases (Mow et al., 1980). Due to the compressibility of the solid matrix pores however, fluid could transport in or out of this biphasic material, allowing changes in volume. Since cartilage (Cohen et al., 1998; Soltz and Ateshian, 2000) and agarose (Huang et al., 2008; Mauck et al., 2000) are known to exhibit a significantly higher modulus in tension than compression, and since this tensioncompression nonlinearity must be taken into account in order to accurately predict experimental outcomes, the solid matrix was modeled as a fiber-reinforced composite having a continuous, random fiber distribution where each fiber bundle is described by a power law (Ateshian et al., 2009), and a ground matrix modeled as a compressible neoHookean solid (Bonet and Wood, 1997). The power law relationship for the individual fiber bundles was described in an earlier study (Ateshian et al., 2009) and the associated material parameters used in the FEM were $\xi$ (a measure of the fiber modulus) and power-law exponent $\alpha$. For the neo-Hookean material, the properties consisted of Young's modulus $E_{Y}$ and Poisson's ratio $v$. The permeability of the porous solid matrix to the interstitial fluid was isotropic and constant, given by $k$. Two of these material parameters were preset: $\alpha=2$ was selected to produce a linear tensile response in the range of small strains, consistent with the known behavior of cartilage; and $v=0$ was selected for the porous ground matrix due to the compressibility of the pore space. The remaining three parameters $\left(\xi, E_{Y}\right.$, and $\left.k\right)$ were obtained by curve-fitting the experimental transient unconfined compression stress-relaxation response of six day 21 MSC-seeded constructs (Fig. 2), using a finite element model that reproduced the geometry of tested disks $(\varnothing 4 \mathrm{~mm}, 1.5 \mathrm{~mm}$ thick, see Mechanical testing of constructs below). The preset value of $v$ was justified based on a sensitivity analysis which demonstrated that the unconfined compression stress-relaxation load response is insensitive to changes in $v$ in the range 0 to 0.15 . Direct measures of the Poisson ratio of acellular $2 \%$ agarose reported a value of 0.12 (Cloyd et al., 2007). To validate these preset and curve-fitted parameters, they were used in finite element analyses that simulated the tensile testing experiments performed on the rectangular constructs. The predicted tensile moduli (extracted from the slope of the finite element analysis stress-strain response) were subsequently compared statistically with the corresponding experimental measurements to verify equivalence and thus validate the curve-fitted parameters.

The finite element analyses for curve-fitting the experimental data in unconfined compression, simulating the tensile response, and modeling the sliding contact between indenter and construct, were performed with the open-source program FEBio available in the public domain (http://www.febio.org), using algorithms valid for biphasic materials undergoing finite deformation (Ateshian et al., 

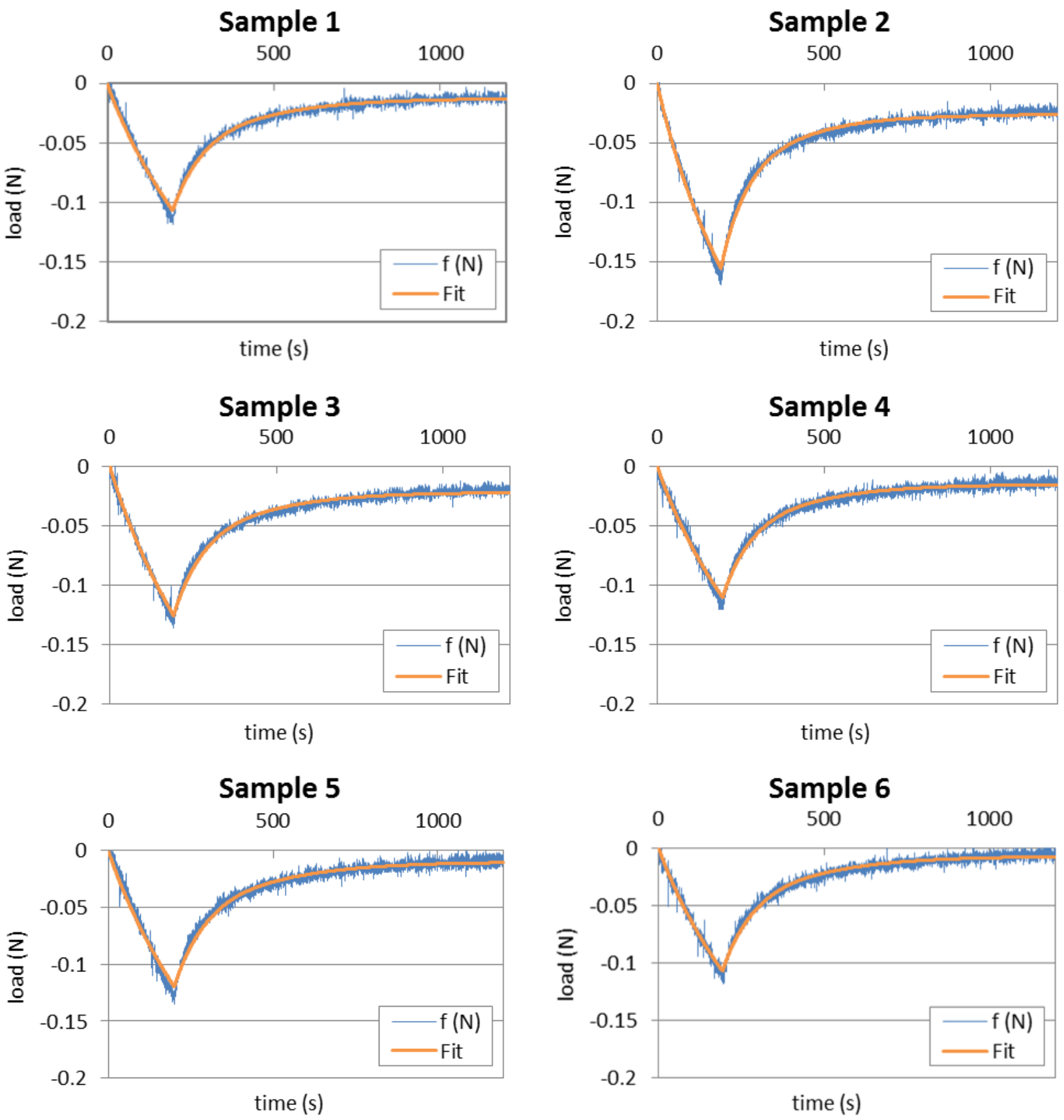

Fig. 2. FEBio curve-fits of experimentally determined stress-relaxation testing from six individual MSC-seeded constructs chondrogenically cultured for $21 \mathrm{~d}$ were used to obtain model parameters for $\xi, E_{Y}$, and $k$.

2010). Curve-fitting was performed using FEBio's builtin least-squares parameter optimization routine, based on the Levenberg-Marquardt algorithm. Sliding contact was modeled using an impermeable spherical indenter with a diameter of $\varnothing 25 \mathrm{~mm}$ sliding over a tissue construct $41.5 \mathrm{~mm}$ long, $7.5 \mathrm{~mm}$ wide, and $1.5 \mathrm{~mm}$ thick. Due to symmetry, only one-half of the geometry was modeled. A reciprocating velocity of $\pm 2.5 \mathrm{~mm} / \mathrm{s}$ was prescribed for the indenter, over a sliding range of $\pm 12.5 \mathrm{~mm}$ centered along the length of the construct. The indenter produced compressive deformation of $20 \%$ of the thickness $(0.3 \mathrm{~mm})$. The contact interface between indenter and construct was assumed to be frictionless, as justified by the observation that the friction coefficient between a smooth impermeable round indenter sliding over native articular is very small, typically on the order of 0.02 (Ateshian and Wang, 1995; Caligaris and Ateshian, 2008); the friction coefficient of cartilage tissue constructs against glass has been observed to be similarly small in our prior studies (Bian et al., 2010b). The bottom of the construct was fixed to a rigid impermeable substrate; this idealized boundary condition was adopted in lieu of the alternative idealized assumption of a frictionless interface with the substrate, based on our experimental observations that the tissue constructs adhered significantly to the substrate. Fluid was allowed to escape from the free boundaries not in direct contact with the indenter. The model consisted of 14208 hexahedral 8-node elements ( 74 along the length, 12 along the width and 16 along the thickness) and 18009 nodes. The mesh was biased along the thickness to produce thinner 
elements near the top and bottom surfaces; a coarser mesh was also employed at the far ends along the length. A series of ten back-and-forth cycles of loading were analyzed, starting with the vertical compressive displacement of the indenter ramped up over $1 \mathrm{~s}$, followed by $5 \mathrm{~s}$ of forward motion and $5 \mathrm{~s}$ of reverse motion, repeated 10 times. FEM results were visualized using the FEBio Postview environment (http://www.febio.org).

\section{Mesenchymal stem cell isolation and culture}

MSCs were isolated from the tibial and femoral bone marrow of 3-6 month old calves (Research 87, Boylston, MA, USA) as previously described (Huang et al., 2009). Cultures were initiated and expanded in high glucose Dulbecco's Modified Eagle's Medium (DMEM) supplemented with $1 \%$ penicillin/streptomycin/fungizone (PSF) and $10 \%$ fetal bovine serum. Cultures were expanded at a ratio of 1:3 through passage two, with media changed twice weekly. MSCs from at least two donors were combined for each study. Separate MSC isolations were carried out per study.

\section{Sliding contact (SLC) loading of acellular and MSC- seeded constructs}

For acellular studies, $2 \%$ and $6 \%$ type VII agarose (Sigma, St Louis, MO, USA) in phosphate-buffered saline (PBS) were separately cast to $1.5 \mathrm{~mm}$ thickness with small porous nylon webs embedded at each end. Gel slabs were then sectioned into rectangular strips $(41.5 \times 7.5 \mathrm{~mm})$. Strips were subjected to $1 \mathrm{~h}$ of SLC at 5, 10 or $20 \%$ axial strain applied at a velocity of $2.5 \mathrm{~mm} / \mathrm{s}$. Additional intact strips were evaluated for tensile properties.

For cell-seeded studies, sterile type VII agarose $\left(49^{\circ} \mathrm{C}\right.$, $4 \% \mathrm{w} / \mathrm{v}$, Sigma, St Louis, MO, USA) in PBS was combined $1: 1$ with MSCs at $25^{\circ} \mathrm{C}$ in chemically defined medium (CM) for a final seeding density of 20 million cells $/ \mathrm{mL}$. CM consisted of DMEM supplemented with $1 \mathrm{X}$ PSF, $0.1 \mu \mathrm{M}$ dexamethasone, $50 \mu \mathrm{g} / \mathrm{mL}$ ascorbate 2-phosphate, $40 \mu \mathrm{g} /$ $\mathrm{mL}$ L-proline, $100 \mu \mathrm{g} / \mathrm{mL}$ sodium pyruvate, $6.25 \mu \mathrm{g} / \mathrm{mL}$ insulin, $6.25 \mu \mathrm{g} / \mathrm{mL}$ transferrin, $6.25 \mathrm{ng} / \mathrm{mL}$ selenous acid, $1.25 \mathrm{mg} / \mathrm{mL}$ bovine serum albumin, and $5.35 \mu \mathrm{g} / \mathrm{mL}$ linoleic acid. Rectangular cell-seeded agarose strips (41.5 x $7.5 \mathrm{~mm}$ ) were formed as above, with porous webbing on either end. Strips were maintained in CM supplemented with $10 \mathrm{ng} / \mathrm{mL}$ TGF- $\beta 3$ (CM+, R\&D Systems, Minneapolis, MN, USA) with media ( $6 \mathrm{~mL} / \mathrm{strip}$ ) changed twice weekly.

After $21 \mathrm{~d}$, mechanical (tensile and compressive) and biochemical properties were evaluated. To determine optimal loading parameters, additional pre-matured ( $21 \mathrm{~d}$ ) strips were subjected to $3 \mathrm{~h}$ of sliding at 5, 10 or $20 \%$ axial strain at $2.5 \mathrm{~mm} / \mathrm{s}(0.1 \mathrm{~Hz})$. Sliding was applied in $\mathrm{CM}$ with $(\mathrm{CM}+)$ or without (CM-) TGF- $\beta 3$, with free-swelling controls cultured in parallel. Gene expression was assessed by real-time PCR directly after sliding. Samples for gene expression were harvested from the loaded region of strips. Two replicate experiments (from two different MSC isolations) were carried out with data from both experiments pooled. Cell viability after loading was assessed using the Live/Dead assay (Molecular Probes, Eugene, OR, USA) for the $20 \%$ axial strain group.
From these preliminary studies, a loading regimen of $20 \%$ axial strain at $2.5 \mathrm{~mm} / \mathrm{s}$ in $\mathrm{CM}+$ media was chosen for subsequent long-term sliding experiments. After $21 \mathrm{~d}$ of pre-culture in $\mathrm{CM}+$, strips were subjected to $21 \mathrm{~d}$ of sliding contact. Loading was applied for $3 \mathrm{~h}$ daily, $5 \mathrm{~d}$ per week with free-swelling controls cultured in parallel. On days 21 and 42, strips were evaluated for tensile and compressive properties, as well as biochemical content and gene expression.

\section{Mechanical testing of constructs}

To evaluate tensile properties, an Instron 5848 Microtester (Instron, Canton, MA, USA) was used to apply uniaxial tension to intact strips seated into 120 grit sandpaper-coated grips as previously described (Huang et al., 2008). Sample dimensions were determined using a digital caliper, and gauge length measured after placement in grips. A $0.5 \%$ / sec strain rate was applied until failure. The ramp tensile modulus was calculated from the measured geometry and the linear region of the stress-strain curve. Ultimate strain (strain at maximum stress) and toughness (integrated area under the stress-strain curve) were also determined for each sample. Preconditioning was not carried out before tensile testing, as previous work has demonstrated that testing cartilage in tension without preconditioning produces highly reproducible results (Park and Ateshian, 2006).

To determine compressive mechanical properties, $\varnothing 4 \mathrm{~mm}$ disks were removed from the loaded region of strips and tested in unconfined compression using a custom testing apparatus (Mauck et al., 2006). A static load of $1.56 \mathrm{kPa}$ was applied to constructs for $5 \mathrm{~min}$ until equilibrium was reached. Following this creep deformation ( 8-10\%), constructs were subjected to $10 \%$ strain (at a ramp rate of $0.05 \% / \mathrm{s}$ ) and then allowed to relax for $1000 \mathrm{~s}$ until equilibrium. Dynamic testing was carried out by applying an additional $1 \%$ sinusoidal deformation to relaxed constructs at a frequency of $1.0 \mathrm{~Hz}$. The equilibrium and dynamic moduli were determined as previously described (Mauck et al., 2006). These data were also used to validate the model parameters chosen by curve-fitting the stress relaxation response and using these extracted parameters to predict the independent tensile testing response of samples (see Results section). After compressive testing, disks were frozen at $-20{ }^{\circ} \mathrm{C}$ for subsequent biochemical evaluation.

\section{Real time polymerase chain reaction (PCR)}

Total RNA was extracted from cores removed from the contact region by two sequential isolations in TRIZOL-chloroform and quantified (ND-1000, Nanodrop Technologies, Wilmington, DE, USA). RNA quality was determined using 260/280 absorption ratios. Reverse transcription and amplification were carried out using an Applied Biosystems 7300 real-time PCR system with intron spanning primers and SYBR Green Reaction Mix (Applied Biosystems, Foster City, CA, USA) as previously described (Huang et al., 2009). Relative expression levels of aggrecan, collagen type II, collagen type I and proteoglycan 4 were quantified using the standard curve method and normalized to glyceraldehyde 3-phosphate dehydrogenase (GAPDH). 


\section{Biochemical analysis}

Papain-digested disks were evaluated for sulfated glycosaminoglycan (GAG) and collagen contents using the 1,9-dimethylmethylene blue dye-binding (Farndale et al., 1986) and orthohydroxyproline (Stegemann and Stalder, 1967) assays, respectively, as in previous studies (Huang et al., 2009). A 1:7.14 orthohydroxyproline:collagen ratio was used (Neuman and Logan, 1950). DNA content was determined using the commercially available PicoGreen dsDNA assay according to the manufacturer's instructions (Molecular Probes, Eugene, OR, USA). GAG and collagen are reported as percentage of construct wet weight while DNA is reported as amount per construct. The wet weights of constructs were highly consistent between groups and across experiments $(p=0.4)$, demonstrating negligible loss of water content due to loading.

\section{Histology and immunohistochemistry}

Constructs were fixed in $4 \%$ paraformaldehyde, dehydrated through a graded series of ethanol washes, and paraffin embedded according to standard protocols. Sections $(8 \mu \mathrm{m})$ were cut onto glass slides and stained with hematoxylin and eosin (H\&E, Sigma, St. Louis, MO, USA), Alcian Blue ( $\mathrm{pH}$ 1.0) or Picrosirius Red for cell distribution, proteoglycans, and collagens, respectively. For immunohistochemistry, antigen retrieval was performed by incubating sections in proteinase $\mathrm{K}(20 \mu \mathrm{g} / \mathrm{mL}$ in TrisEDTA buffer, $\mathrm{pH} 8.0$ ) at $37^{\circ} \mathrm{C}$ for $15 \mathrm{~min}$, then at $25^{\circ} \mathrm{C}$ for $10 \mathrm{~min}$. Primary antibodies specific for collagen type I (MAB3391, Millipore, Billerica, MA, USA) and collagen type II (11-116B3, Developmental Studies Hybridoma Bank, Iowa City, IA, USA) were used. Subsequent reaction and visualization with DAB chromagen reagent (DAB150 IHC Select, Millipore, Billerica, MA) was carried out as described in detail in previous studies (Huang et al., 2008). Colour images were captured at $2.5 \mathrm{x}$ or $10 \mathrm{x}$ magnification using a microscope equipped with a colour CCD digital camera and the QCapturePro acquisition software.

Depth-dependent inhomogeneity in matrix deposition was assessed on Alcian Blue-stained construct crosssections (Image J, National Institute of Health, Bethesda, MD, USA). To carry out these analyses, color images were converted to 32-bit grayscale images. Each cross-section was divided into three layers of equal thickness (surface, middle, deep), and mean intensity was measured in each layer (255: black, 0: white).

\section{Statistical analysis}

Statistical analysis was performed on mechanical, biochemical and histological data using one way ANOVA ( $n=6$ for all studies). A two-way ANOVA was used to assess differences in gene expression, with independent variables of axial strain and media condition. Where significance was indicated by ANOVA, Fisher's LSD post-hoc tests were performed. Mean values for gene expression were determined from four to six samples per experimental group. Significance for all statistical measures was determined at $p \leq 0.05$ and a trend toward significance noted when $p<0.1$. All values are reported as mean \pm standard deviation.

\section{Results}

\section{Sliding contact bioreactor characterization}

A displacement-controlled bioreactor was constructed to apply sliding contact (SLC) to hydrogel constructs by means of spherical indenters (Fig. 1A-D). Indenter sizes ranging from $\varnothing 2-\varnothing 25 \mathrm{~mm}$ were initially considered. Calculations derived from the indenter geometry (spherical) and an assumed axial deformation of $10 \%$ showed that increasing indenter diameter increased the contact area, while decreasing the strain rate for a given sliding velocity (Fig. 1E). As indenter curvature was less steep with increasing diameter, axial strain rates were minimized with larger indenter sizes. Assuming an indenter size of $\varnothing 25 \mathrm{~mm}$ and $10 \%$ axial strain, sliding velocities of 2.5-20 mm/s were next considered. Axial strain rates generated at $20 \mathrm{~mm} / \mathrm{s}$ reached nearly $200 \% / \mathrm{s}$ while lower sliding velocities generated much lower strain rates $(\sim 25$ $50 \% / \mathrm{s}$, Fig. 1F). From these initial calculations, an indenter diameter size of $\varnothing 25 \mathrm{~mm}$ and sliding velocity of $2.5 \mathrm{~mm} / \mathrm{s}$ were incorporated into the bioreactor design and used for all experiments. Given a sliding contact path of $\sim 13 \mathrm{~mm}$ in length, the frequency of sliding was $\sim 0.1 \mathrm{~Hz}$.

In addition to the above considerations, a range of axial strains was also applied to assess the effects of SLC on chondrogenesis. Calculations based on $\varnothing 25 \mathrm{~mm}$ indenter size and $2.5 \mathrm{~mm} / \mathrm{s}$ sliding velocity showed that increasing the applied axial strain (5-20\%) also increased indenter contact area (Fig. 1G). Axial strain rate was also higher with increasing axial strain, with non-uniform strain rates generated throughout the displaced region. While strain rates were lowest at the center of the indented region (regardless of the applied strain), strain rates increased with increasing distance from the center due to the curvature of the indenter (Fig. 1H).

\section{Finite element modeling (FEM) of sliding contact}

Curve-fitting of the unconfined compression stressrelaxation response of day $21 \mathrm{MSC}$-seeded constructs produced the following properties: $E_{Y}=11.1 \pm 5.5 \mathrm{kPa}, \xi=$ $19.5 \pm 3.5 \mathrm{kPa}$, and $k=(22.5 \pm 2.2) \times 10^{-3} \mathrm{~mm}^{4} / \mathrm{N} \times \mathrm{s}($ mean \pm standard deviation, $n=6$ samples). The coefficient of determination for these curve-fits was $R^{2}=0.984 \pm 0.002$ (Fig. 2). When these properties were used to simulate the tensile responses of rectangular constructs at a rate of $0.5 \% / \mathrm{s}$, the resulting predicted tensile modulus was $303 \pm 57 \mathrm{kPa}$. Experimental measurements of the tensile modulus for similar rectangular constructs produced 337 $\pm 104 \mathrm{kPa}$ (see below), a value not statistically different from the predicted modulus $(p=0.4)$. This verification provides a validation of the tissue constitutive model and fitted properties, since measurements obtained from one experimental test have been used to successfully predict the outcome of another experimental test.

Next, a finite element analysis was performed to estimate the magnitude and distribution of mechanical signals imparted by SLC on pre-matured, MSC-seeded constructs, using these validated properties (Figs. 3 and 4). The analysis predicted the response to ten reciprocal cycles of sliding, representing the first $100 \mathrm{~s}$ of loading, providing representative measures of the construct response to 

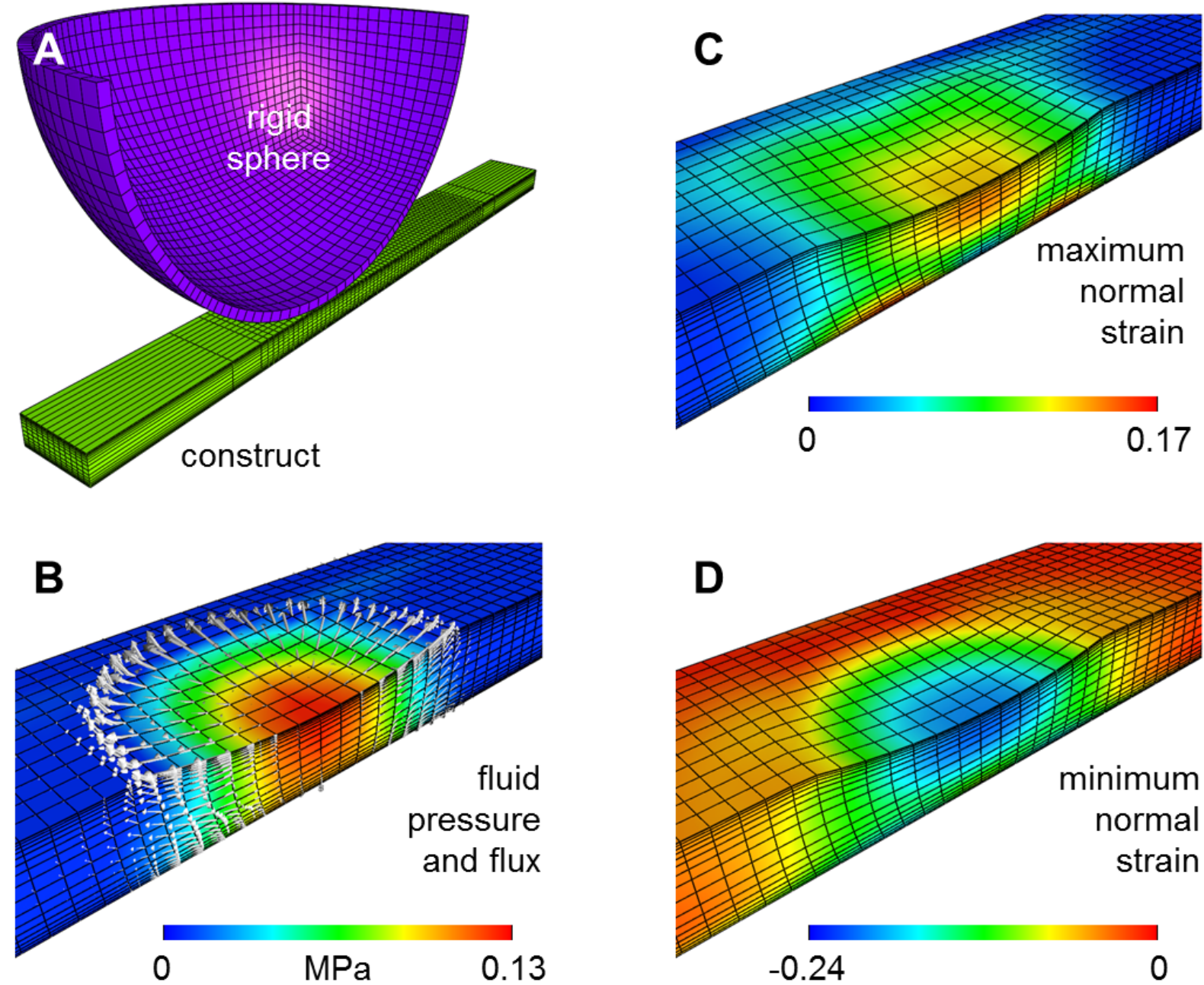

Fig. 3. (A) Finite element model of spherical indenter sliding over the tissue construct. Due to symmetry, only onehalf of the actual geometry was modeled. (B) Fluid pressure distribution (color map) and fluid flux vectors (arrows) at time $\mathrm{t}=97.5 \mathrm{~s}$ after initiation of loading (indenter is hidden). (C) Maximum normal strain and (D) minimum normal strain at the same time point.

sliding contact. The analysis of these ten cycles required $15 \mathrm{~h}$ of computational time. The spatial distribution of the interstitial fluid flux and pressure are reported in Fig. 3, on the return stroke of the last cycle, when the indenter reached the center of the construct $(t=97.5 \mathrm{~s})$. The temporal response of these variables over the ten cycles of loading is also reported at each of the elements depicted in Fig. 4A, depicting the extent by which these variables underwent relaxation over $100 \mathrm{~s}$ of sliding contact.

This analysis showed that fluid pressure (Fig. 4B) was highest directly underneath the indenter (element 1) and nearly uniform through the thickness of the construct (Fig. 3B). The peak value of the pressure at element 1 was approximately $0.15 \mathrm{MPa}$ and relaxed down to $0.12 \mathrm{MPa}$ after the tenth cycle (Fig. 4B); based on a simple exponential decay, the characteristic time constant of this relaxation was approximately $110 \mathrm{~s}$, and it may be estimated by extrapolation that the steady-state value of the pressure would be approximately $0.1 \mathrm{MPa}$. The maximum normal strain was tensile throughout the construct (Fig. 3C); its magnitude was elevated in the top half of the thickness under the center of the contact region, where its principal direction was oriented tangential to the contact surface. An elevated value was also observed at the interface with the substrate, likely due to the assumption of adhesive conditions at that boundary. The maximum normal strain also exhibited a relaxation behavior, reducing in magnitude over time (Fig. 4C). The minimum normal strain was compressive throughout the construct, with its peak magnitude located directly under the indenter, and decreasing in magnitude with depth from the surface (Fig. 3D), consistent with previous calculations (Fig. 1G). Over time, the peak magnitude of this compressive strain held relatively constant at element 1 , at -0.20 (Fig. 4D).

Regions of highest fluid flow were observed at the outer edge of the contact region, producing efflux from the construct (Fig. 3B). Despite the longitudinal motion of the indenter, fluid flux flowed radially out from underneath the indenter at all locations around the edge of contact, including the leading and trailing edges. Though the greatest fluid flux magnitude occurred at the surface ( $\sim 0.004 \mathrm{~mm} / \mathrm{s}$ peak value), elevated values were observed throughout the thickness of the construct, underneath the footprint of the contact region. However, fluid flux remained negligible near the outer boundaries of the construct (Fig. 3B). 

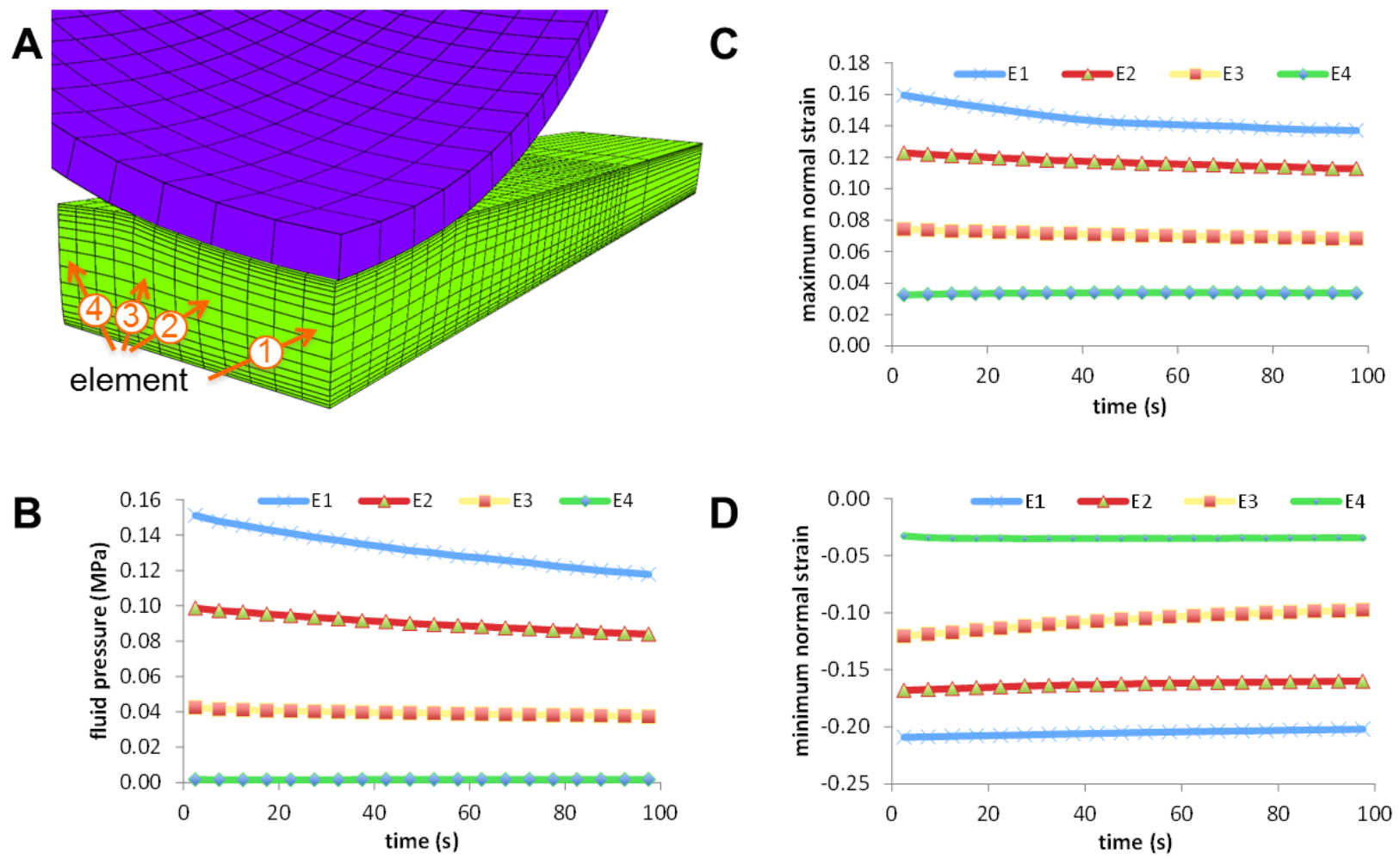

Fig. 4. (A) A temporal response is provided at four selected elements (E1 through E4) located halfway along the sliding path of the indenter, spanning the width of the construct, approximately halfway through the thickness. (B) The interstitial fluid pressure, $(\mathbf{C})$ maximum normal strain and (D) minimum normal strain are reported at the time points when the spherical indenter passes over the elements, reflecting ten back-and-forth cycles. A slow decay in the magnitude of these variables is observed over the $100 \mathrm{~s}$ analysis time.

\begin{abstract}
Validation of sliding contact bioreactor
After construction of the bioreactor system, initial studies were performed on acellular agarose constructs. Consistent with our previous studies (Huang et al., 2008), increasing agarose concentration resulted in a higher tensile modulus, without changing the ultimate strain (Fig. 5A). For both $2 \%$ and $6 \%$ acellular agarose constructs, ultimate tensile strain was $\sim 10 \%$. Under chondrogenic culture, the tensile modulus of MSC-seeded constructs was higher at day 21 (compared to $2 \%$ agarose starting values) and was akin to that of the $6 \%$ acellular agarose constructs $(\sim 270 \mathrm{kPa})$. The ultimate strain also improved by day 21 to $\sim 40 \%$ as matrix was deposited within the construct (compared to $\sim 10 \%$ starting values, Fig. $5 \mathrm{~A}$ ). Similarly, the compressive properties and biochemical content increased with time in culture (Fig. 5B). Under SLC, both $2 \%$ and $6 \%$ acellular gels consistently fractured through the midline (along the sliding contact path) for all axial strains applied (not shown). Fracture was observed in all cases within a few minutes after initiation of loading. In contrast, pre-cultured MSC-seeded constructs remained intact after $3 \mathrm{~h}$ of SLC at $20 \%$ axial strain (and with much longer loading regimens, as detailed below). No adverse effect on cell viability was observed with SLC compared to free-swelling (FS) controls (Fig. 5C). Based on these findings, MSC-seeded constructs were chondrogenically pre-cultured for $21 \mathrm{~d}$ prior to the initiation of sliding for subsequent studies.
\end{abstract}

\section{Chondrogenic gene expression in MSC-seeded constructs with short-term application of sliding contact}

To assess the effects of SLC on chondrogenic gene expression, loading was applied for $3 \mathrm{~h}$ at $2.5 \mathrm{~mm} / \mathrm{s}$ on MSC-seeded constructs following $21 \mathrm{~d}$ of pre-culture. Axial strains of $5 \%, 10 \%$ and $20 \%$ were evaluated and SLC was carried out in defined media in the presence $(\mathrm{CM}+)$ and absence (CM-) of TGF- $\beta 3$. When SLC was applied in CM-, collagen type II and aggrecan expression were higher in groups subjected to $20 \%$ axial strain, compared to FS. An axial strain-dependent effect was observed in SLC constructs in $\mathrm{CM}+$. While $5 \%$ strain did not alter collagen type II or aggrecan expression compared to FS controls $(p>0.1)$, SLC at $10 \%$ axial compression increased the expression of both collagen type II $(p=0.015)$ and aggrecan (trend, $p=0.067$ ). Expression levels for these genes were further increased when SLC was applied at $20 \%$ strain, and were significantly higher compared to all other groups within each media condition (Fig. 6). An increase in proteoglycan 4 expression was only observed in SLC constructs at $20 \%$ strain in $\mathrm{CM}+$. Interestingly, compared to the chondrogenic genes, collagen type I gene expression was considerably less responsive to SLC (Fig. 6D). As results from these studies demonstrated that SLC applied at $20 \%$ axial strain in CM+ was optimal for promoting chondrogenic gene expression, these parameters were employed for subsequent long-term studies. 
A

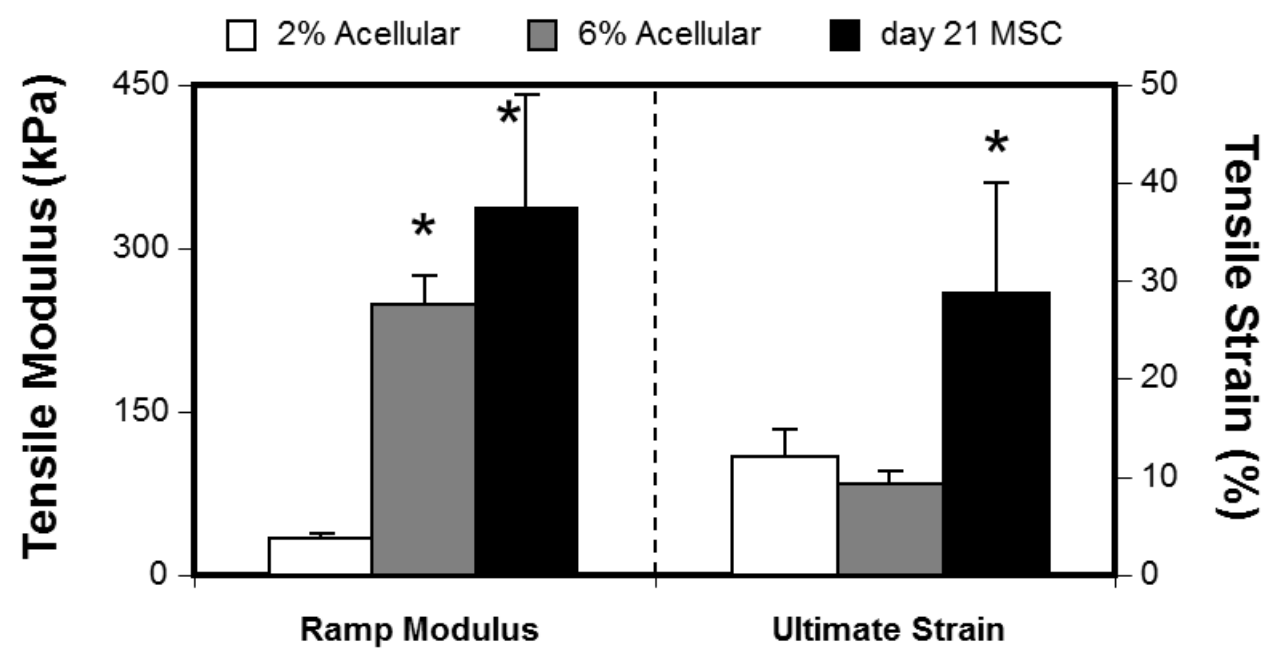

B

\begin{tabular}{|l|c|c|c|}
\hline Construct Properties & Value & Coaded & Free-swelling \\
\hline Compressive modulus $(\mathrm{kPa})$ & $13.6 \pm 5.5$ & \\
\hline Tensile modulus $(\mathrm{kPa})$ & $337 \pm 104$ \\
\hline DNA content $(\mu \mathrm{g} / \mathrm{construct)}$ & $1.45 \pm 0.2$ \\
\hline GAG content $(\% \mathrm{ww})$ & $1.09 \pm 0.2$ \\
\hline Collagen content $(\% \mathrm{ww})$ & $0.11 \pm 0.04$ \\
\hline
\end{tabular}

Fig. 5. Functional properties of acellular and MSC-seeded agarose constructs. (A) While the tensile modulus increased with higher agarose concentration, the ultimate strain was comparable between $2 \%$ and $6 \%$ acellular agarose. After $21 \mathrm{~d}$ of culture in $\mathrm{CM}+$, the tensile modulus of MSC-seeded constructs was the same as $6 \%$ agarose, but the ultimate strain was 4-fold higher compared to acellular constructs. (B) Mechanical and biochemical properties of MSCseeded constructs improved with time in chondrogenic culture. (C) Preliminary studies showed no adverse effect on cell viability when SLC was applied to pre-cultured MSC-seeded constructs at $20 \%$ axial strain at $2.5 \mathrm{~mm} / \mathrm{s}$ for $3 \mathrm{~h}$. Data represent the mean and standard deviation of six samples. * indicates greater than $2 \%$ agarose $(p<0.05)$.

Functional properties of MSC-seeded constructs after long-term application of sliding contact

To evaluate whether long-term application of SLC could direct functional maturation, MSC-seeded constructs were subjected to $21 \mathrm{~d}$ of daily sliding contact ( $20 \%$ axial strain, $2.5 \mathrm{~mm} / \mathrm{sec}$ sliding velocity, $0.1 \mathrm{~Hz}, 3 \mathrm{~h} / \mathrm{d}$ ) following $21 \mathrm{~d}$ of pre-culture in $\mathrm{CM}+$. By day 42 , tensile properties were significantly greater compared to day 21 , for both FS and SLC groups $(p<0.05)$. The tensile stiffness and tensile modulus both increased with SLC, reaching values of $\sim 0.8 \mathrm{~N} / \mathrm{mm}$ and $\sim 750 \mathrm{kPa}$, respectively (compared with $\sim 0.6 \mathrm{~N} / \mathrm{mm}$ and $\sim 600 \mathrm{kPa}$ for FS controls, Fig. 7A, B). Day 21 and day 42 values for ultimate tensile strain were similar; this measure was unchanged with SLC and remained at $\sim 40 \%$ (not shown).

DNA content was maintained at day 21 starting values with long-term SLC; in contrast the DNA content of FS samples continued to increase past day 21 values (Fig. 7C). When normalized to wet weight, both GAG and collagen contents were higher in SLC compared to FS (trend $p<0.1$ ).
This difference between FS and SLC was more pronounced when GAG and collagen contents were normalized to DNA, with $p$ values of 0.058 and 0.001 , respectively (not shown). Although GAG content plateaued in FS between days 21 and 42 , collagen content was higher by day $42(p<0.01$, Fig. 7D, E). Collectively, these results demonstrated that repeated SLC loading induced a shift from cell proliferation to enhanced matrix deposition, resulting in an increase in tensile properties.

Depth-dependent matrix distribution in MSC-seeded constructs after long-term sliding contact

To assess whether matrix distribution was affected by SLC, cross-sections were stained with Alcian Blue to visualize proteoglycans (Fig. 8A, B), and staining intensity was quantified through the depth. Histology showed distinct differences in depth-dependent matrix distribution at day 42. Alcian blue staining intensity was comparable in the deep zones of FS and SLC constructs. While there was a slight increase in intensity at the surface zones of FS 

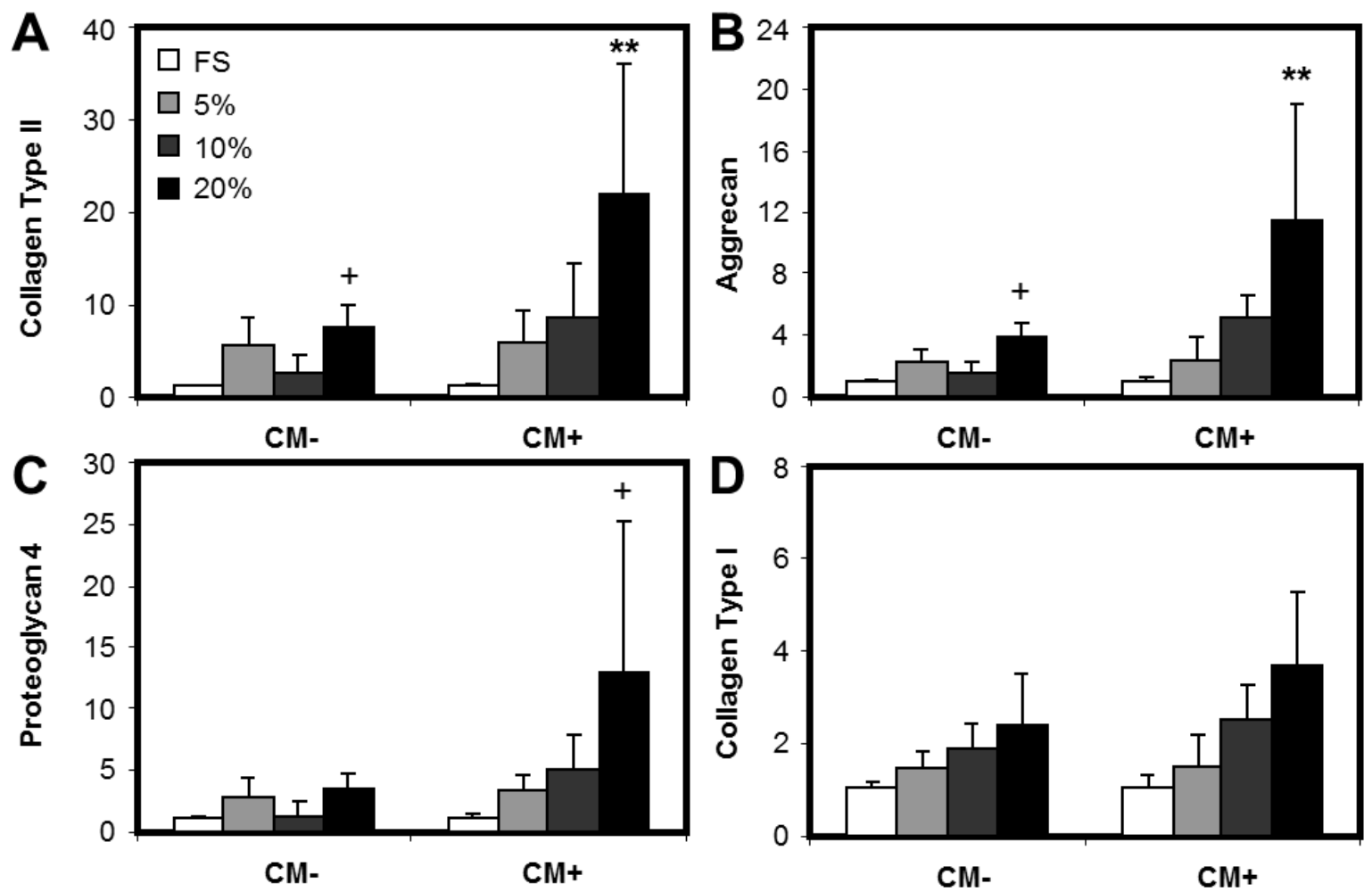

Fig. 6. Cartilaginous gene expression with short-term application of SLC. (A) Collagen type II, (B) aggrecan, (C) proteoglycan 4 and (D) collagen type I gene expression was dependent on both media condition and axial strain. For all chondrogenic genes, expression was highest in $\mathrm{CM}+$ with $20 \%$ axial strain. No significant difference was observed in collagen type I expression with SLC. Gene expression (normalized to GAPDH) was normalized to free-swelling controls within media type. ${ }^{* *}$ indicates greater than all within media condition $(p<0.05),+$ indicates greater than free-swelling control within media condition $(p<0.05)$. Data represent the mean and standard deviation of 4-6 samples per group per time point.

constructs, the staining intensity at the surface of SLC constructs was greater. Proteoglycan distribution was homogeneous through the depth in FS, however, type II collagen staining was non-uniform. The surface half of FS constructs exhibited even staining while the deep regions were marked by punctate type II collagen staining. In contrast, type II collagen was continuously distributed through the depth in SLC constructs; as with Alcian Blue staining, intense collagen staining was observed in the surface region (Fig. 8C).

\section{Discussion}

MSCs are an attractive cell type for cartilage regeneration therapies given their chondrogenic potential; however, the mechanical properties of MSC-seeded constructs remain limited to date. To improve these functional properties and instill the structural features of articular cartilage, we and others have previously explored direct mechanical stimulation to enhance MSC chondrogenesis (Angele et al., 2004; Grad et al., 2011; Huang et al., 2010b; Mouw et al., 2007). Within a variety of biomaterial systems, MSCs are sensitive to their mechanical environment and MSC differentiation and functional properties can be enhanced by mechanical conditioning. However, current systems to date are not specifically designed to instill hallmark properties of articular cartilage (inhomogeneity and anisotropy). As these characteristic properties arise during cartilage maturation with the advent of physiologic loading (Williamson et al., 2003b), we hypothesized that a loading configuration more representative of joint loading may be required.

To design such a system, we first considered the native mechanical cues arising from joint movement. During physiologic loading, most of the applied load is supported by pressurization of the interstitial fluid, which shields the solid matrix from excessive deformation. Direct measurements of interstitial fluid pressure in cartilage, and the ability to accurately predict such measurements with the biphasic theory by incorporating the tension-compression nonlinearity of the solid matrix, have established that intrinsic (flow-independent) viscoelasticity of the solid matrix plays a significantly lesser role in the dissipative part of the cartilage response (Park and Ateshian, 2006; Soltz and Ateshian, 1998; Soltz and Ateshian, 2000). Theoretical predictions suggest that the fluid load support can be sustained for several hours of loading under congruent, migrating contact configurations, as long as the migrating speed is significantly greater than the characteristic diffusion velocity of interstitial fluid (Ateshian and Wang, 1995; Caligaris and Ateshian, 2008). The same phenomenon is observed for higher indenter deformation (given a relatively large indenter radius), 

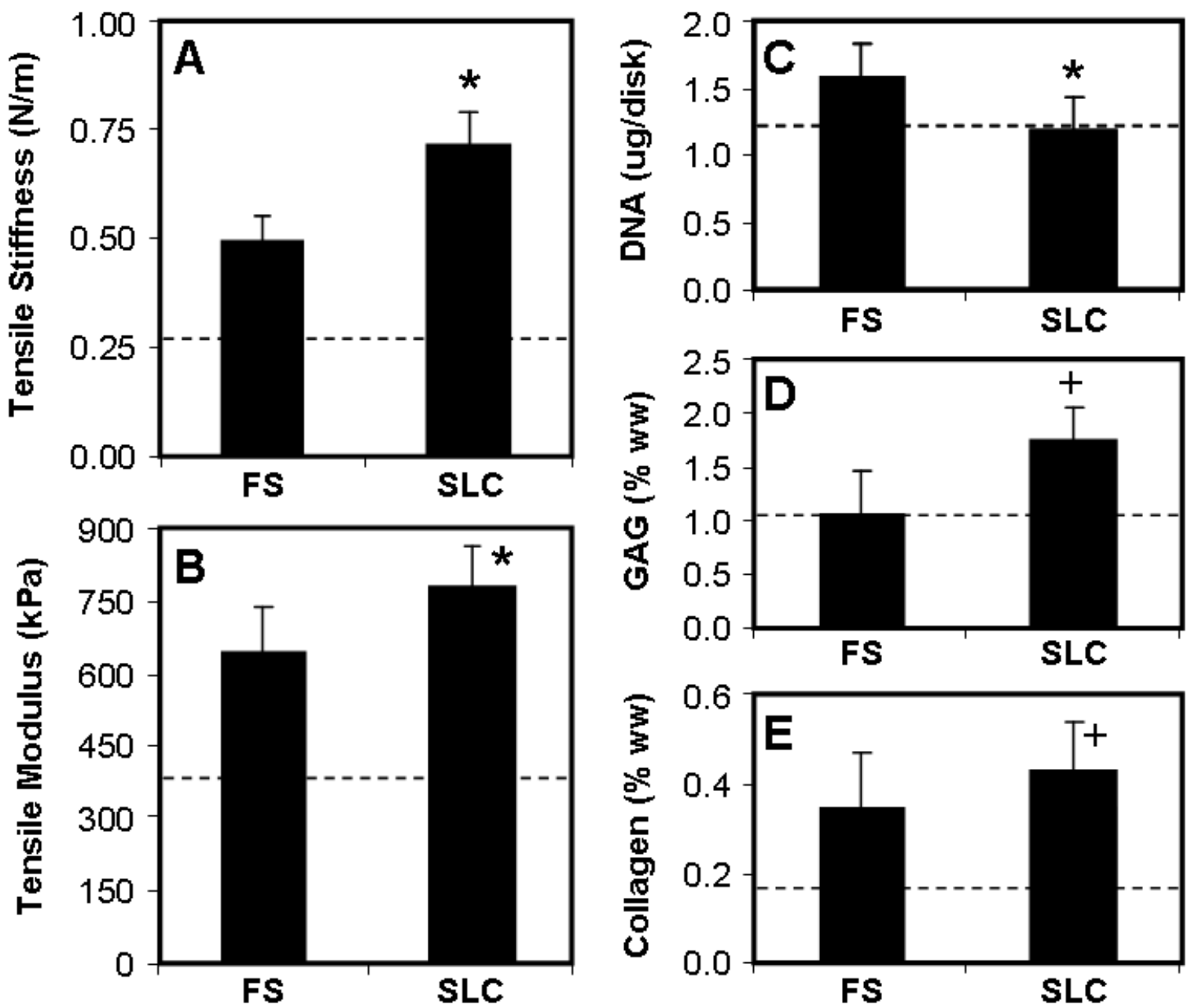

Fig. 7. Mechanical and biochemical properties of MSC-seeded constructs after long-term application of SLC. (A) Tensile stiffness and (B) tensile modulus increased after $21 \mathrm{~d}$ of SLC. (C) DNA content ( $\mu \mathrm{g} /$ construct) was lower for SLC compared to day 42 FS. Bulk measures of (D) GAG and (E) collagen content (\% wet weight) were higher with SLC compared to FS at day 42.* indicates greater than day 42 controls $(p<0.05),+$ indicates trend toward greater than day 42 controls $(p<0.1)$. Data represent mean and standard deviation of six samples per group. Dashed line represents day 21 starting values.

as this also leads to greater contact areas that extend the time required for pressurized fluid to escape from the loaded regions. This sustained fluid pressurization occurs only when the contact area migrates over the porous tissue construct, since sustained loading over a stationary contact area would cause relatively rapid fluid efflux and loss of pressurization. With these considerations in mind, we designed a novel bioreactor system with spherical indenters and a reciprocating contact area. Calculations were performed varying the indenter diameter, the travel velocity and the applied axial deformation to assess contact areas and strain rates, in order to best replicate in vivo conditions, given the evolving properties of the growing tissue constructs.

Applying these considerations, we constructed and characterized a novel sliding contact bioreactor and evaluated short-term mechanosensitivity and longterm development of functional properties in MSCseeded agarose constructs. Initial short term loading studies showed that chondrogenic gene expression was dependent on the applied axial deformation and TGF- $\beta 3$ supplementation. Aggrecan and collagen type II gene expression was enhanced under $20 \%$ axial deformation with both media conditions, but was greatest in the presence of TGF- $\beta 3$. This was consistent with our previous studies showing that long-term improvements in functional chondrogenesis was only observed in media containing TGF- $\beta 3$ (Huang et al., 2010a). Interestingly, human MSC-seeded fibrin-polyurethane sponges subjected to simultaneous compression and shear responded positively to loading only in the absence of TGF- $\beta 1$ (Li et al., 2009a). While chondrogenic gene expression was higher in groups treated with TGF- $\beta 1$, no additional effect was observed with loading when exogenous TGF- $\beta 1$ was present. Our different findings may be due to a number of factors, including the difference in pre-culture time allowed prior to the initiation of loading ( $7 \mathrm{~d}$ ) or the type of biomaterial used. MSCs within a fibrin-polyurethane composite may deposit matrix molecules at a different rate than MSCs encapsulated within agarose. The elaboration of a welldispersed and connected ECM has important consequences for the translation of mechanical signals by cells; we and 
A

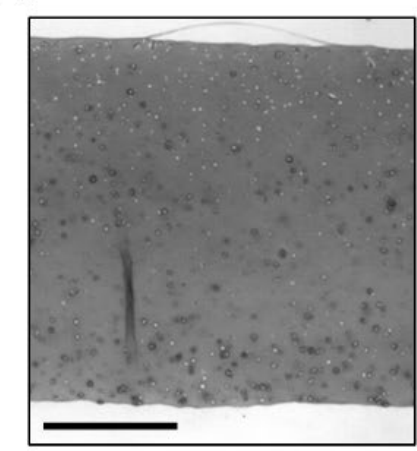

SLC

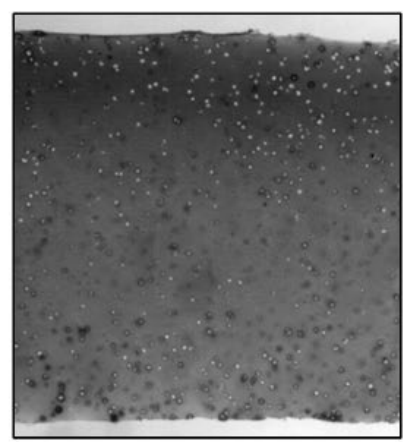

B

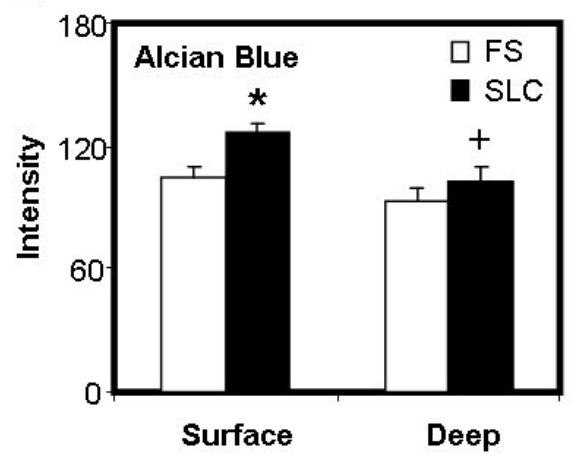

255 (black)

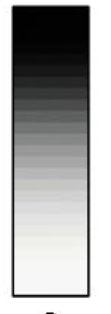

0

C
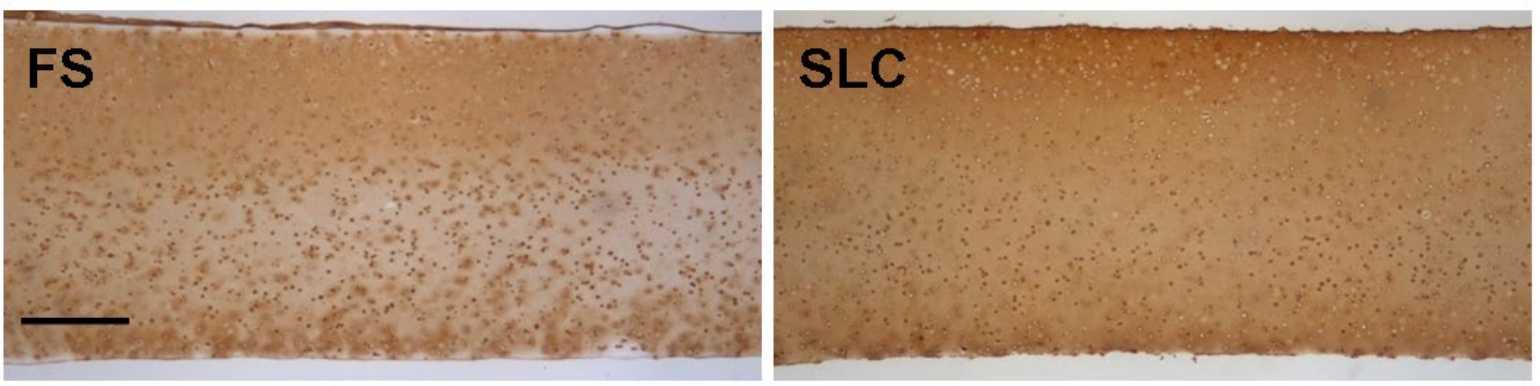

Fig. 8. Depth-dependent distribution of ECM in MSC-seeded constructs after long-term application of SLC. (A) Grayscale images of Alcian Blue-stained cross-sections were assessed for zonal heterogeneity. (B) Intensity of Alcian Blue staining at the surface zones increased with SLC. Deep zone staining intensity was not different between groups. (C) Collagen type II was more continuously distributed in SLC constructs compared to FS controls, combined with more intense staining in the surface region. * indicates higher than all values $(p<0.05)$. Data represent mean and standard deviation of six samples per group. Scale bar: $0.5 \mathrm{~mm}$.

others have previously shown that dynamic compression applied after 0 or $21 \mathrm{~d}$ of pre-culture within agarose results in dramatically different functional outcomes (Huang et al., 2010a; Thorpe et al., 2010). In addition, the bioreactor system used in the studies of Li et al. (2009a) is distinct from the current system, in that shear loading is superimposed on dynamic compressive loading. Though not characterized or described in their study, the interaction of physical signals imparted by their bioreactor and the local microenvironment surrounding MSCs within their heterogeneous scaffold material at the time of loading are likely to be very different compared to the system used in the current work.

Based on our short-term studies, we next applied long-term sliding contact using optimized parameters (20\% axial deformation with $10 \mathrm{ng} / \mathrm{mL}$ TGF- $\beta 3$ ). With 3 weeks of SLC loading, tensile properties and biochemical content of MSC-based constructs improved compared to non-loaded controls. We also observed biochemical inhomogeneity within the constructs subjected to sliding, with collagen type II staining most intense in the surface region. Unexpectedly, inhomogeneity in proteoglycan distribution mirrored that of collagen, where PG staining intensity was greatest in the surface region with SLC. This finding is inconsistent with the structure of native articular cartilage, where proteoglycans are greatest in the middle and deep zones, and so suggests that sliding contact may have a general stimulatory effect in this surface region. Indeed, in cartilage explants, aggrecan expression is highest in regions of highest fluid flow (Buschmann et al., 1999). FEM analysis showed that the physical signals arising from sliding contact were indeed depth-dependent. Tensile and compressive strains and fluid flux in particular were consistently elevated near the surface regions, though elevated values of fluid pressure and fluid flow also spanned the construct depth. The tensile strains generated with sliding also exhibited direction-dependence with tensile strains of $>10 \%$ observed parallel to the sliding direction. Therefore, while SLC does impart mechanical signals that share some features with dynamic compressive loading, the overall mechanical environment generated by SLC is considerably more complex and uniquely depth dependent. Interestingly, preliminary experiments with acellular agarose constructs showed that fracturing along the midline (parallel to the sliding direction) occurred soon after the initiation of sliding contact. While the tensile moduli of acellular constructs differed, the ultimate tensile strains were consistently $\sim 10 \%$. As proof of principle, day 21 MSC-based constructs (ultimate tensile strain: $\sim 40 \%$ ) were able to withstand hours of repeated loading, suggesting that the physical cues predicted by the FEM analysis were reflected in our sliding contact system, and that the complex mechanical environment generated by sliding contact loading may dictate the depth dependent localization of matrix molecules observed in this study.

Our FEM predicts that the mechanical environment experienced by the cells in the surface half of constructs is complex. Tensile and compressive strains are both present, along with elevated fluid pressure and radially directed fluid flow. While chondrocyte (Buschmann et al., 1995; Lima et 
al., 2007; Mauck et al., 2002; Mauck et al., 2000) and MSC (Huang et al., 2010a; Huang et al., 2004a; Mauck et al., 2007; Mouw et al., 2007; Thorpe et al., 2008) response to dynamic compressive strains is relatively well documented, fewer studies have assessed the effect of tensile strains in a cartilage tissue engineering context. In general, dynamic compressive loading enhances chondrogenic gene expression and, in the case of chondrocytes, improves GAG content. Previous work applying dynamic tensile loading to chondrocyte-seeded fibrin showed reduced proteoglycan synthesis with loading (Connelly et al., 2004). Tensile loading of superficial zone chondrocytes seeded in fibrin promoted proteoglycan synthesis, while the same loading applied to deep zone chondrocytes did not (Vanderploeg et al., 2008). When cyclic tension was applied to MSCs seeded in collagen-GAG scaffolds, the rate of GAG synthesis was enhanced (McMahon et al., 2008). In another study using MSC-seeded fibrin gels, proteoglycan synthesis was only enhanced during early stages of tensile loading while protein synthesis remained elevated at later stages (Connelly et al., 2010); collagen type I expression was also stimulated while no change was observed in collagen type II or aggrecan expression, consistent with a fibrocartilaginous phenotype. Although we observed a small increase in collagen type I expression with sliding contact, we saw a far greater response in collagen type II and aggrecan expression levels. Collectively, these studies show that both compressive and tensile deformations applied to MSC-seeded constructs can enhance GAG and collagen content, consistent with the results of the current study.

While the tensile properties and collagen content improved with sliding contact by $25 \%$ and $15 \%$, respectively, the values reached are still far lower than that of articular cartilage. Because the collagen content was relatively low, collagen orientation was not readily assessed by polarized light microscopy and preliminary analysis of collagen alignment showed no differences between groups. It is possible that 3 weeks of sliding was insufficient and longer-term loading will be required to enhance collagen anisotropy. Alternative methods of analysis, such as Fourier transform infrared imaging spectroscopy (Bi et al., 2005) or second harmonic imaging microscopy (Stoller et al., 2002), may also be used to capture early changes in collagen alignment that were not evident from polarized light microscopy analysis. In this study, tensile properties were only assessed parallel to the sliding direction; however these properties could also be assessed perpendicular to the sliding direction to evaluate the development of mechanical anisotropy. Further, it would be of interest to evaluate the depth-dependent inhomogeneity that may arise from differential matrix deposition through the construct (Kelly et al., 2006; Wang et al., 2002).

While we chose a 3 week pre-culture duration based on our previous studies using dynamic compression, a longer pre-culture duration may enhance baseline construct properties prior to the initiation of sliding and lead to improved mechanical outcomes, as tensile properties continue to increase past this time point (Huang et al., 2008). In addition, differences in construct geometries slow the rate of matrix accumulation over time, as the diffusion limitations in the large rectangular agarose strips used in this study would be expected to be more severe compared to smaller agarose cylinders used in previous studies. To overcome these limitations, dynamic culture conditions (agitation of media) may be investigated in future studies. In addition, the presence of agarose itself may limit the extent of matrix remodeling as agarose is biologically inert and does not degrade. The morphology of chondrocytes in the superficial zone of native cartilage is flattened in shape and oriented parallel to the surface; the use of agarose may prevent cells from adopting such morphology. As a rounded phenotype is necessary for proper chondrogenic induction, a hydrogel that evolves with time in culture may be more ideal. Recent work suggests that hydrogels containing hydrolytically or matrix metalloproteinase (MMP)-degradable components enhance collagen deposition by increasing pore-size over time (Bryant and Anseth, 2003; Chung et al., 2009). Such novel biomaterials, coupled with bioreactors systems that provide defined and spatially regulated mechanical cues, may better direct MSC-mediated matrix remodeling and construct maturation in response to sliding contact. Ultimately, better recapitulation of the direction dependent mechanical properties that typify native tissues, and result from its adaptation to a complex loading environment, may improve the long term success of engineered constructs.

\section{Acknowledgements}

This work was supported by the National Institutes of Health for RLM (EB008722, AR053668) and GAA (R01GM083925), the Penn Center for Musculoskeletal Disorders, and Graduate Research Fellowships from the National Science Foundation (AHH and BMB). The type II collagen antibody developed by Thomas F. Linsenmayer was obtained from the Developmental Studies Hybridoma Bank developed under the auspices of the NICHD and maintained by the University of Iowa, Department of Biology, Iowa City, IA 52242, USA. We wish to confirm that there are no known conflicts of interest associated with this publication and there has been no significant financial support for this work that could have influenced its outcome.

\section{References}

Akizuki S, Mow VC, Muller F, Pita JC, Howell DS, Manicourt DH (1986) Tensile properties of human knee joint cartilage: I. Influence of ionic conditions, weight bearing, and fibrillation on the tensile modulus. J Orthop Res 4: 379-392.

Albro MB, Chahine NO, Li R, Yeager K, Hung CT, Ateshian GA (2008) Dynamic loading of deformable porous media can induce active solute transport. J Biomech 41: 3152-3157.

Angele P, Schumann D, Angele M, Kinner B, Englert C, Hente R, Fuchtmeier B, Nerlich M, Neumann C, Kujat R (2004) Cyclic, mechanical compression enhances 
chondrogenesis of mesenchymal progenitor cells in tissue engineering scaffolds. Biorheology 41: 335-346.

Archer CW, Dowthwaite GP, Francis-West PH (2003) Development of synovial joints. Birth Defects Res C 69: 144-155.

Ateshian GA, Hung CT (2003) Functional properties of native articular cartilage. In: Functional Tissue Engineering (F. Guilak, D.L. Butler, S.A. Goldstein, D.J. Mooney, eds), Springer-Verlag, New York, pp 46-68.

Ateshian GA, Wang H (1995) A theoretical solution for the frictionless rolling contact of cylindrical biphasic articular cartilage layers. J Biomech 28: 1341-1355.

Ateshian GA, Warden WH, Kim JJ, Grelsamer RP, Mow VC (1997) Finite deformation biphasic material properties of bovine articular cartilage from confined compression experiments. J Biomech 30: 1157-1164.

Ateshian GA, Rajan V, Chahine NO, Canal CE, Hung CT (2009) Modeling the matrix of articular cartilage using a continuous fiber angular distribution predicts many observed phenomena. J Biomech Eng 131: 061003.

Ateshian GA, Maas S, Weiss JA (2010) Finite element algorithm for frictionless contact of porous permeable media under finite deformation and sliding. J Biomech Eng 132: 061006.

Athanasiou KA, Rosenwasser MP, Buckwalter JA, Malinin TI, Mow VC (1991) Interspecies comparisons of in situ intrinsic mechanical properties of distal femoral cartilage. J Orthop Res 9: 330-340.

Athanasiou KA, Zhu CF, Wang X, Agrawal CM (2000) Effects of aging and dietary restriction on the structural integrity of rat articular cartilage. Ann Biomed Eng 28: 143-149.

Bi X, Li G, Doty SB, Camacho NP (2005) A novel method for determination of collagen orientation in cartilage by Fourier transform infrared imaging spectroscopy (FT-IRIS). Osteoarthritis Cartilage 13: 10501058.

Bian L, Fong JV, Lima EG, Stoker AM, Ateshian GA, Cook JL, Hung CT (2010a) Dynamic mechanical loading enhances functional properties of tissue-engineered cartilage using mature canine chondrocytes. Tissue Eng Part A 16: 1781-1790.

Bian L, Ng K, Lima E, Xu D, Jayabalan P, Ateshian G, Stoker A, Cook J, Hung C (2010b) Applied dynamic loading enhances mechanical properties of engineered cartilage using adult chondrocytes. Trans Orthop Res 35: 0382 .

Bonet J, Wood RD (1997) Nonlinear continuum mechanics for finite element analysis. Cambridge University Press, New York, Chapter 5, pp 117-143.

Bryant SJ, Anseth KS (2003) Controlling the spatial distribution of ECM components in degradable PEG hydrogels for tissue engineering cartilage. J Biomed Mater Res A 64: 70-79.

Buschmann MD, Gluzband YA, Grodzinsky AJ, Hunziker EB (1995) Mechanical compression modulates matrix biosynthesis in chondrocyte/agarose culture. J Cell Sci 108: 1497-1508.

Buschmann MD, Kim YJ, Wong M, Frank E, Hunziker EB, Grodzinsky AJ (1999) Stimulation of aggrecan synthesis in cartilage explants by cyclic loading is localized to regions of high interstitial fluid flow. Arch Biochem Biophys 366: 1-7.

Caligaris M, Ateshian GA (2008) Effects of sustained interstitial fluid pressurization under migrating contact area, and boundary lubrication by synovial fluid, on cartilage friction. Osteoarthritis Cartilage 16: 1220-1227.

Chahine NO, Albro MB, Lima EG, Wei VI, Dubois CR, Hung CT, Ateshian GA (2009) Effect of dynamic loading on the transport of solutes into agarose hydrogels. Biophys J 97: 968-975.

Charlebois M, McKee MD, Buschmann MD (2004) Nonlinear tensile properties of bovine articular cartilage and their variation with age and depth. J Biomech Eng 126: 129-137.

Chung C, Beecham M, Mauck RL, Burdick JA (2009) The influence of degradation characteristics of hyaluronic acid hydrogels on in vitro neocartilage formation by mesenchymal stem cells. Biomaterials 30: 4287-4296.

Cloyd JM, Malhotra NR, Weng L, Chen W, Mauck RL, Elliott DM (2007) Material properties in unconfined compression of human nucleus pulposus, injectable hyaluronic acid-based hydrogels and tissue engineering scaffolds. Eur Spine J 16: 1892-1898.

Cohen B, Lai WM, Mow VC (1998) A transversely isotropic biphasic model for unconfined compression of growth plate and chondroepiphysis. J Biomech Eng 120: 491-496.

Connelly JT, Vanderploeg EJ, Levenston ME (2004) The influence of cyclic tension amplitude on chondrocyte matrix synthesis: experimental and finite element analyses. Biorheology 41: 377-387.

Connelly JT, Vanderploeg EJ, Mouw JK, Wilson C, Levenston ME (2010) Tensile loading modulates BMSC differentiation and the development of engineered fibrocartilage constructs. Tissue Eng Part A 16: 1913-1923.

Erickson IE, Huang AH, Chung C, Li RT, Burdick JA, Mauck RL (2009) Differential maturation and structurefunction relationships in mesenchymal stem cell- and chondrocyte-seeded hydrogels. Tissue Eng Part A 15: 1041-1052.

Farndale RW, Buttle DJ, Barrett AJ (1986) Improved quantitation and discrimination of sulphated glycosaminoglycans by use of dimethylmethylene blue. Biochim Biophys Acta 883: 173-177.

Grad S, Lee CR, Gorna K, Gogolewski S, Wimmer MA, Alini M (2005) Surface motion upregulates superficial zone protein and hyaluronan production in chondrocyte-seeded three-dimensional scaffolds. Tissue Eng 11: 249-256.

Grad S, Gogolewski S, Alini M, Wimmer MA (2006a) Effects of simple and complex motion patterns on gene expression of chondrocytes seeded in 3D scaffolds. Tissue Eng 12: 3171-3179.

Grad S, Lee CR, Wimmer MA, Alini M (2006b) Chondrocyte gene expression under applied surface motion. Biorheology 43: 259-269.

Grad S, Eglin D, Alini M, Stoddart MJ (2011) Physical stimulation of chondrogenic cells in vitro: a review. Clin Orthop Relat Res 469: 2764-2772.

Grad S, Loparic M, Peter R, Stolz M, Aebi U, Alini M (2012) Sliding motion modulates stiffness and friction 
coefficient at the surface of tissue engineered cartilage. Osteoarthritis Cartilage 20: 288-295.

Huang CY, Hagar KL, Frost LE, Sun Y, Cheung HS (2004a) Effects of cyclic compressive loading on chondrogenesis of rabbit bone-marrow derived mesenchymal stem cells. Stem Cells 22: 313-323.

Huang CY, Reuben PM, D'Ippolito G, Schiller PC, Cheung HS (2004b) Chondrogenesis of human bone marrow-derived mesenchymal stem cells in agarose culture. Anat Rec A Discov Mol Cell Evol Biol 278: 428436.

Huang AH, Yeger-McKeever M, Stein A, Mauck RL (2008) Tensile properties of engineered cartilage formed from chondrocyte- and MSC-laden hydrogels. Osteoarthritis Cartilage 16: 1074-1082.

Huang AH, Stein A, Tuan RS, Mauck RL (2009) Transient exposure to TGF-beta3 improves the mechanical properties of MSC-laden cartilage constructs in a density dependent manner. Tissue Eng Part A 15: 3461-3472.

Huang AH, Farrell MJ, Kim M, Mauck RL (2010a) Long-term dynamic loading improves the mechanical properties of chondrogenic mesenchymal stem cell-laden hydrogel. Eur Cell Mater 19: 72-85.

Huang AH, Farrell MJ, Mauck RL (2010b) Mechanics and mechanobiology of mesenchymal stem cell-based engineered cartilage. J Biomech 43: 128-136.

Kelly TA, Ng KW, Wang CC, Ateshian GA, Hung CT (2006) Spatial and temporal development of chondrocyteseeded agarose constructs in free-swelling and dynamically loaded cultures. J Biomech 39: 1489-1497.

Kempson GE (1982) Relationship between the tensile properties of articular cartilage from the human knee and age. Ann Rheum Dis 41: 508-511.

Kempson GE (1991) Age-related changes in the tensile properties of human articular cartilage: a comparative study between the femoral head of the hip joint and the talus of the ankle joint. Biochim Biophys Acta 1075: 223-230.

Li Z, Kupcsik L, Yao SJ, Alini M, Stoddart MJ (2009a) Mechanical load modulates chondrogenesis of human mesenchymal stem cells through the TGF-beta pathway. J Cell Mol Med 14: 1338-1346.

Li Z, Yao SJ, Alini M, Stoddart MJ (2009b) Chondrogenesis of human bone marrow mesenchymal stem cells in fibrin-polyurethane composites is modulated by frequency and amplitude of dynamic compression and shear stress. Tissue Eng Part A 16: 575-584.

Lima EG, Bian L, Ng KW, Mauck RL, Byers BA, Tuan RS, Ateshian GA, Hung CT (2007) The beneficial effect of delayed compressive loading on tissue-engineered cartilage constructs cultured with TGF-beta3. Osteoarthritis Cartilage 15: 1025-1033.

Mankin HJ, Mow VC, Buckwalter JA, Iannotti JP, Ratcliffe A(1994) Form and function of articular cartilage. In: Orthopaedic Basic Science (S.R. Simon, ed), American Academy of Orthopaedic Surgeons, Rosemont, pp 1-44.

Mauck RL, Soltz MA, Wang CC, Wong DD, Chao PH, Valhmu WB, Hung CT, Ateshian GA (2000) Functional tissue engineering of articular cartilage through dynamic loading of chondrocyte-seeded agarose gels. J Biomech Eng 122: 252-260.
Mauck RL, Seyhan SL, Ateshian GA, Hung CT (2002) Influence of seeding density and dynamic deformational loading on the developing structure/function relationships of chondrocyte-seeded agarose hydrogels. Ann Biomed Eng 30: 1046-1056.

Mauck RL, Hung CT, Ateshian GA (2003) Modeling of neutral solute transport in a dynamically loaded porous permeable gel: implications for articular cartilage biosynthesis and tissue engineering. J Biomech Eng 125: 602-614.

Mauck RL, Yuan X, Tuan RS (2006) Chondrogenic differentiation and functional maturation of bovine mesenchymal stem cells in long-term agarose culture. Osteoarthritis Cartilage 14: 179-189.

Mauck RL, Byers BA, Yuan X, Tuan RS (2007) Regulation of cartilaginous ECM gene transcription by chondrocytes and MSCs in 3D culture in response to dynamic loading. Biomech Model Mechanobiol 6: 113125.

McMahon LA, Reid AJ, Campbell VA, Prendergast PJ (2008) Regulatory effects of mechanical strain on the chondrogenic differentiation of MSCs in a collagen-GAG scaffold: experimental and computational analysis. Ann Biomed Eng 36: 185-194.

Mouw JK, Connelly JT, Wilson CG, Michael KE, Levenston ME (2007) Dynamic compression regulates the expression and synthesis of chondrocyte-specific matrix molecules in bone marrow stromal cells. Stem Cells 25: 655-663.

Mow VC, Guo XE (2002) Mechano-electrochemical properties of articular cartilage: their inhomogeneities and anisotropies. Annu Rev Biomed Eng 4: 175-209.

Mow VC, Kuei SC, Lai WM, Armstrong CG (1980) Biphasic creep and stress relaxation of articular cartilage in compression? Theory and experiments. J Biomech Eng 102: $73-84$.

Muir H (1980) The chemistry of the ground substance of joint cartilage. In: The Joints and Synovial Fluid (L. Sokoloff, ed), Academic Press, New York, pp 27-94.

Neuman RE, Logan MA (1950) The determination of hydroxyproline. J Biol Chem 184: 299-306.

Park S, Ateshian GA (2006) Dynamic response of immature bovine articular cartilage in tension and compression, and nonlinear viscoelastic modeling of the tensile response. J Biomech Eng 128: 623-630.

Roth V, Mow VC (1980) The intrinsic tensile behavior of the matrix of bovine articular cartilage and its variation with age. J Bone Joint Surg Am 62: 1102-1117.

Soltz MA, Ateshian GA (1998) Experimental verification and theoretical prediction of cartilage interstitial fluid pressurization at an impermeable contact interface in confined compression. J Biomech 31: 927-934.

Soltz MA, Ateshian GA (2000) Interstitial fluid pressurization during confined compression cyclical loading of articular cartilage. Ann Biomed Eng 28: 150159.

Stegemann H, Stalder K (1967) Determination of hydroxyproline. Clin Chim Acta 18: 267-273.

Stoddart MJ, Ettinger L, Hauselmann HJ (2006) Enhanced matrix synthesis in de novo, scaffold free 
cartilage-like tissue subjected to compression and shear. Biotechnol Bioeng 95: 1043-1051.

Stoller P, Reiser KM, Celliers PM, Rubenchik AM (2002) Polarization-modulated second harmonic generation in collagen. Biophys J 82: 3330-3342.

Thorpe SD, Buckley CT, Vinardell T, O’Brien FJ, Campbell VA, Kelly DJ (2008) Dynamic compression can inhibit chondrogenesis of mesenchymal stem cells. Biochem Biophys Res Commun 377: 458-462.

Thorpe SD, Buckley CT, Vinardell T, O’Brien FJ, Campbell VA, Kelly DJ (2010) The response of bone marrow-derived mesenchymal stem cells to dynamic compression following TGF-beta3 induced chondrogenic differentiation. Ann Biomed Eng 38: 2896-2909.

Vanderploeg EJ, Wilson CG, Levenston ME (2008) Articular chondrocytes derived from distinct tissue zones differentially respond to in vitro oscillatory tensile loading. Osteoarthritis Cartilage 16: 1228-1236.

Wang CC, Deng JM, Ateshian GA, Hung CT (2002) An automated approach for direct measurement of twodimensional strain distributions within articular cartilage under unconfined compression. J Biomech Eng 124: 557567.

Williamson AK, Chen AC, Sah RL (2001) Compressive properties and function-composition relationships of developing bovine articular cartilage. J Orthop Res 19: 1113-1121.

Williamson AK, Chen AC, Masuda K, Thonar EJ, Sah RL (2003a) Tensile mechanical properties of bovine articular cartilage: variations with growth and relationships to collagen network components. J Orthop Res 21: 872880 .

Williamson AK, Masuda K, Thonar EJ, Sah RL (2003b) Growth of immature articular cartilage in vitro: correlated variation in tensile biomechanical and collagen network properties. Tissue Eng 9: 625-634.

Woo SL,Akeson WH, Jemmott GF (1976) Measurements of nonhomogeneous, directional mechanical properties of articular cartilage in tension. J Biomech 9: 785-791.

\section{Discussion with Reviewers}

Reviewer I: Can the authors discuss whether they believe such bioreactor systems will be used clinically, or if they feel their real utility lies in improving our basic understanding of MSC/cartilage mechanobiology?

Authors: There exists a competing duality in the field of tissue engineering between what is 'practical' for clinical application, and what might be 'necessary' in terms of design to fully recapitulate articular structure and function. With the former consideration, any added level of complication would increase cost, time, and regulatory hurdles to the tissue engineering process. For the latter, imperfect replication (or evolution) of native tissue structure and function would increase the probability of early construct failure. It has not yet been ascertained by the field whether one needs to fully replicate the native tissue structure and function prior to implantation of an engineered construct. The bioreactor system employed here promotes development of key features in cartilage that are not yet established (i.e., tensile modulus). It may be that, when situated in a native loading environment, a construct would undergo the same maturation process as in our bioreactor system. If true, then our system could serve to optimize this maturation process by defining salubrious rehabilitation regimens by helping us to understand the underlying mechanobiology of this loading modality. Alternatively, if the field ultimately finds that any tissue that does not match native tissue properties is doomed to failure, then our device may be used to promote a clinically relevant construct for implantation, albeit at an added cost.

Reviewer I: This study demonstrated significant differences in gene expression due to loading in the short-term, yet only a trend towards changes in ECM accumulation in the long-term. What does this say about utility of RT-PCR for predicting longer term outcomes in bioreactor studies?

Authors: Our general feeling on this matter is that RTPCR is best used as a rough guide of the direction in which a construct is going with respect to the loading conditions applied, and that measurement of real content, accumulated over days and/or weeks, represents the most valid culmination of this response. While it is sometimes frustrating to wait such lengthy periods, we often find disagreement between early term anabolic responses and long term functional maturation. This was most telling in our recent publication (Huang et al., 2010a), where dynamic compressive loading could lead to increased anabolic gene expression response in the short term, but a generally catabolic response when applied over several weeks, most likely due to degradative processes that are simultaneously initiated.

Reviewer II: Given the heterogeneity of the mechanical microenvironment of cells within tissue engineered constructs, to what extent can models which are based on homogenization techniques and bulk mechanical properties of tissues predict the mechanical signals at the cell level and what promising alternatives are available to improve our evaluation of these signals?

Authors: This is an important point to consider in the interpretation of any change in cellular behavior in $3 \mathrm{D}$ culture in response to a defined mechanical stimulus. Even in relatively simple loading configurations (i.e., dynamic axial unconfined compression), there exist marked gradients in fluid pressure and fluid flow through the radial expanse of a construct. Here, with sliding contact, we show marked spatial heterogeneity in the kinds (and magnitudes) of physical signals that cells will experience. This does suggest that methods that assume a homogeneous response across a tissue construct would fail to capture the complexity of cellular response based on local signals. Methods pioneered by the Grodzinsky lab at MIT showed that radioisotope labeling, coupled with histology, could localize and quantify proteoglycan and collagen production in the cellular microenvironment (for example, Buschmann et al., 1999). Work by Schinagl et al. (1997) and Wang et al. (2002) developed methods for 
local analysis of construct material properties, which could further inform theoretical representations and predication of local biophysical response with construct development. Others have separated constructs into annular and core regions with loading to try to isolate local responses. We have also experimented with the use of reporter promoter constructs (luciferase and GFP as the readout) to visualize and quantify local cell behavior (Mauck et al., 2007). We have recently adopted newer methods, including laser capture microdissection and quantitative fluorescent in situ hybridization (Raj et al., 2008) to query individual cell response. As molecular (and even whole genome) analysis methods improve, enabling quantification at the single cell level, new insights might be gleaned as to the true local response of a cell to its complex, but well defined, biophysical environment.

\section{Additional References}

Schinagl RM, Gurskis D, Chen AC, Sah RL (1997) Depth-dependent confined compression modulus of fullthickness bovine articular cartilage. J Orthop Res 15: 499-506.

Raj A, van den Bogaard P, Rifkin SA, van Oudenaarden A, Tyagi S (2008) Imaging individual mRNA molecules using multiple singly labeled probes. Nat Methods 5: 877879. 